\title{
Photometry of $2006 \mathrm{RH}_{120}$ : an asteroid temporary captured into a geocentric orbit ${ }^{\star}$
}

\author{
T. Kwiatkowski ${ }^{1}$, A. Kryszczyńska ${ }^{1}$, M. Polińska ${ }^{1}$, D. A. H. Buckley ${ }^{2}$, D. O’Donoghue ${ }^{2}$, P. A. Charles ${ }^{2}$, \\ L. Crause ${ }^{2}$, S. Crawford ${ }^{2}$, Y. Hashimoto ${ }^{2}$, A. Kniazev ${ }^{2}$, N. Loaring ${ }^{2}$, E. Romero Colmenero ${ }^{2}$, \\ R. Sefako ${ }^{2}$, M. Still ${ }^{2}$, and P. Vaisanen ${ }^{2}$ \\ 1 Astronomical Observatory, Adam Mickiewicz University, Słoneczna 36, 60-286 Poznań, Poland \\ e-mail: tkastr@amu.edu.pl \\ 2 South African Astronomical Observatory, Observatory Road, Observatory 7925, South Africa
}

Received 15 September 2008 / Accepted 12 December 2008

\section{ABSTRACT}

\begin{abstract}
Aims. From July 2006 to July 2007 a very small asteroid orbited the Earth within its Hill sphere. We used this opportunity to study its rotation and estimate its diameter and shape.

Methods. Due to its faintness, $2006 \mathrm{RH}_{120}$ was observed photometrically with the new 10-m SALT telescope at the SAAO (South Africa). We obtained data on four nights: 11, 15, 16, and 17 March 2007 when the solar phase angle remained almost constant at $74^{\circ}$. The observations lasted about an hour each night and the object was exposed for 7-10 s through the "clear" filter.

Results. From the lightcurves obtained on three nights we derived two solutions for a synodical period of rotation: $P_{1}=1.375 \pm$ $0.001 \mathrm{~min}$ and $P_{2}=2.750 \pm 0.002 \mathrm{~min}$. The available data are not sufficient to choose between them. The absolute magnitude of the object was found to be $H=29.9 \pm 0.3 \mathrm{mag}$ (with the assumed slope parameter $G=0.25$ ) and its effective diameter $D=2-7 \mathrm{~m}$, depending on the geometric albedo $p_{V}$ (with the most typical near-Earth asteroids albedo $p_{V}=0.18$ its diameter would be $D=$ $3.3 \pm 0.4 \mathrm{~m})$. The body has an elongated shape with the $a / b$ ratio greater than 1.4. It probably originates in low-eccentricity Amor or Apollo orbits. There is still a possibility, which needs further investigation, that it is a typical near-Earth asteroid that survived the aerobraking in the Earth's atmosphere and returned to a heliocentric orbit similar to that of the Earth.
\end{abstract}

Key words. techniques: photometric - minor planets, asteroids

\section{Introduction}

Among Earth-approaching asteroids there are two groups of objects which - instead of passing the Earth - can spend some time in its vicinity. This behaviour is interesting from the point of view of orbital dynamics, but also presents a good opportunity for their detailed study with telescopes and radar. What is more, the low relative velocity of some of them make them good targets for sample recovery missions.

The first group consists of the so-called Earth co-orbitals. The semiaxes of their orbits lie in the interval $[0.99,1.01]$ AU which triggers the resonance with Earth. Over one revolution about the Sun, their orbits are close to Keplerian but in the longer term small perturbations from the Earth induce very specific motion. In a frame co-rotating with the Earth they perform double librations about the $\mathrm{L}_{4}$ and $\mathrm{L}_{5}$ Lagrange points, moving along the Earth's orbit on a "horseshoe" (HS) path. When they approach the Earth in the gap of the horseshoe, they bounce back or on rare occasions - transfer to the quasi-satellite (QS) orbit in the vicinity of the Earth (see Mikkola et al. 2006, Fig. 1). The QS episode can last from several to thousands of years during which the asteroid moves on open loops near the Earth. After that it jumps back to the HS orbit (Mikkola et al. 2006). QS asteroids are not gravitationally bound to the Earth. These objects, albeit close to the planet, are outside its Hill sphere and are not true satellites. One of the Earth co-orbitals on the QS orbit

* Based on observations made with the Southern African Large Telescope (SALT) was $2003 \mathrm{YN}_{107}$, which stayed within $0.1 \mathrm{AU}$ of the Earth for nine years. Another example is $2004 \mathrm{GU}_{9}$, our current quasisatellite (Connors et al. 2004).

The second group of asteroids move on extremely Earth-like orbits and their motion is dominated by close approaches with the Earth. They do not experience a 1:1 resonance with the planet but every synodic period they approach it with small relative velocities and can potentially be captured by its gravity (Brasser \& Wiegert 2008).

The first such asteroid was $1991 \mathrm{VG}$, which became a temporary satellite of the Earth in 1991 (Tancredi 1998). It was captured by its gravitation but most of the open loop it made around the planet was outside its Hill sphere. The photometric observations of this 5-10 m object gave contradicting results (IAUC 5402) and it was not possible to decide whether it was a natural body or space debris.

J002E3, the second known object to orbit the Earth, was discovered in 2002. It came from the heliocentric orbit through the $\mathrm{L}_{1}$ Lagrange point, spent a year inside the Earth's Hill sphere orbiting it six times on an open-loop orbit and then left the EarthMoon system. One of the parameters of the orbital fit, the area to mass ratio (AMR), was found to be typical of artificial satellites (http: //neo.jpl .nasa.gov/news/news 136.html, last accessed 2008-09-01). The analysis of the past history of J002E3 indicated it might have visited the Earth-Moon system in 1971 and be a Saturn IV-B - the third stage of one of the Apollo program rocket boosters (Lambert et al. 2004). 
To check the hypothesis of an artificial origin, spectroscopic observations of J002E3 were performed both in the visible and near-IR ranges. A strong correlation of the absorption features in the near-IR with the spectra of white paint containing titanium-oxide has been found (Jorgensen et al. 2003). The high-resolution visible spectra of J002E3 were also compared to spectra of space-weathered rockets, showing a good agreement (Lambert et al. 2004).

An interesting attempt to model the photometric behaviour of J002E3 was also made by Lambert et al. (2004). Using five lightcurves from different observing geometries they derived a spin vector, the angle of precession and the absolute size of the cylinder that best matched observations. This allowed them to confirm that the observed body had the dimensions of the Saturn IV B. Even though the low quality of the photometric data and a simplified method of analysis make their results preliminary, their approach can be used for other similar objects. The shapes of the largest rocket boosters are well known and it is relatively easy to predict their brightness variations for the purpose of checking the origin of the unknown objects approaching the Earth with small relative velocities.

The situation of J002E3 repeated three years later, when on 14 September 2006 a new near-Earth object was found by the Catalina Sky Survey. It turned out that this several metres in diameter body was moving on a geocentric orbit which suggested it was space debris. As a result it was given a designation 6R10DB9 and classified as a Distant Artificial Satellite.

In December 2006 new astrometric observations made it possible to compute a more accurate orbit of 6R10DB9 showing it entered the Earth's Hill sphere in July 2006, was moving on an open-loop orbit and would leave the Hill sphere in July 2007. Its perigee distances were 2.2 LD (11 Sep. 2006), 1.4 LD (3 Jan. 2007), 0.9 LD (25 Mar.) and 0.7 LD (14 Jun.), where LD stands for a lunar distance. Contrary to J002E3, the AMR was found to be much smaller than typical values for artificial satellites (Bill Gray, personal communication). This raised suspicion that 6R10DB9 could actually be a natural body and not a burned-out rocket booster; this generated interest among observers.

When approaching the 25 March perigee, on the suggestion of Carl Hergenrother, 6R10DB9 was observed photometrically with the SALT telescope (results of these observations are reported in this paper). Attempts to obtain its spectrum with the 6.5 MMT telescope failed (Carl Hergenrother, personal communication). Fortunately, its small distance from the Earth in midJune allowed it to be observed by the Goldstone radar (Lance Benner, private communication). The results of those observations made it possible to finally confirm the natural origin of 6R10DB9 (Hergenrother et al., in preparation). In February 2008 it was given a provisional designation $2006 \mathrm{RH}_{120}$ (Bressi et al. 2008).

\section{Observations}

Due to its faintness and possible short period of light variations, photometric observations of $2006 \mathrm{RH}_{120}$ had to be performed with a large telescope. Fortunately, we could use for this purpose the new $10 \mathrm{~m}$ Southern African Large Telescope (SALT) located at the South African Astronomical Observatory (IAU code B31) near Sutherland. At that time the instrument was still in the commissioning and performance verification phase and so not all telescope subsystems were operational.

The telescope, which is closely modelled on the HobbyEberly Telescope (Ramsey et al. 1998) works similarly to the
Table 1. Aspect data for $2006 \mathrm{RH}_{120}$.

\begin{tabular}{cccccc}
\hline \hline $\begin{array}{c}\text { Date } \\
2007\end{array}$ & $r$ & $\Delta$ & $\begin{array}{c}\text { Phase } \\
\text { angle }\end{array}$ & $\begin{array}{c}\lambda \\
(\mathrm{J} 2000)\end{array}$ \\
$(\mathrm{UT})$ & $(\mathrm{AU})$ & $(\mathrm{AU})$ & $\left(^{\circ}\right)$ & $\left(^{\circ}\right)$ & $\left({ }^{\circ}\right)$ \\
\hline Mar. 11.87 & 0.995 & 0.0058 & 74.7 & 105.9 & -52.4 \\
Mar. 15.85 & 0.996 & 0.0047 & 74.2 & 104.5 & -37.1 \\
Mar. 16.84 & 0.996 & 0.0044 & 74.3 & 104.2 & -31.9 \\
Mar. 17.81 & 0.996 & 0.0041 & 74.4 & 104.0 & -26.2 \\
\hline
\end{tabular}

Note: Due to its small geocentric distance, topocentric coordinates related to IAU station B31 (SALT) were used: $\lambda$ and $\beta$ are the ecliptic longitude and latitude, while $r$ and $\Delta$ are the distances of the asteroid from the Sun and the observer, respectively.

Arecibo radio telescope: it has a spherical primary mirror, which does not move during the observation, and a prime focus payload with a spherical aberration corrector and science instruments, mounted on a "Tracker" which follows objects across the sky.

The primary mirror consists of 91 hexagonal mirror segments, $1.2 \mathrm{~m}$ corner-to-corner, which are designed to be kept aligned by a closed-loop active optics system. The principal axis of the primary has a fixed tilt of $37^{\circ}$ with respect to zenith and the whole telescope is only rotated about the vertical axis for object acquisition. As a result, SALT can observe targets within the annular region on the sky with a zenith distance from $31^{\circ}$ to $43^{\circ}$ and the accessible declination range is $-75^{\circ}<\delta<+10^{\circ}$. Prior to the observation, SALT is slewed to the appropriate azimuth and stops there. When the object enters the annular region of accessibility, it is tracked using the Tracker and data are collected for about one hour (this time can be longer or shorter, depending on the declination). More details about SALT can be found in Buckley (2001), Buckley et al. (2006), O’Donoghue et al. (2006) and references therein.

In the imaging mode, SALT uses the SALTICAM camera consisting of a mosaiced pair of $2048 \times 4102$ E2V 44-82 CCDs, with $15 \mu \mathrm{m}$ square pixels. During our observations we used it in a $4 \times 4$ binning mode, which - with SALTICAM's $f / 2$ focal ratio - gave us a plate scale of 0.56 per pixel. This also resulted in shortened read-out times of $15 \mathrm{~s}$. The science field of view of SALTICAM has a diameter of $8^{\prime}$ which helped when observing a fast moving target.

SALT works in a queue-scheduling mode, where observations are planned in advance and executed by SALT operators and astronomers. In the case of targets of opportunity, a PI can submit a proposal by e-mail, updating positions every night if need be - this was the mode of operation used for our observations.

During the commissioning period the delivered point spread function of the telescope was not optimal, due to a combination of image quality problems arising from optical alignment issues, coupled with the not yet operational active optics control of the primary mirror array. Because of this when describing the observing conditions, we do not report the seeing but rather the average $F W H M$ of the stellar images, measured directly on the CCD frames.

We planned to observe $2006 \mathrm{RH}_{120}$ in March, as soon as it became visible in the dark Moon time. The window of opportunity for SALT started on 10 March and lasted till 18 March, during which the object was becoming brighter and its sky movement faster. At the same time it was approaching the Milky Way which made the observations more difficult. The observing geometry during this period is presented in Table 1 . 
The first observations of $2006 \mathrm{RH}_{120}$, performed on 11 March, were considered partly as a test. The weather was rather poor with some scattered clouds present and the average $F W H M$ of stellar images was $2^{\prime \prime}$. According to the ephemeris, the target should have about $V=21 \mathrm{mag}$ and to avoid trailed images we had to expose for only $10 \mathrm{~s}$. The first series of images were obtained with a clear filter, but due to some stray light from the tracker autocollimator (operating at $670 \mathrm{~nm}$ ) we changed to the $V$ filter.

The pre-reduced data were made available for analysis after two days. It appeared that the unfiltered frames, despite the stray light, gave better results and showed signs of a several minutes long rotation period. The $V$ filter frames had much lower $\mathrm{S} / \mathrm{N}$ and at minimum light the asteroid was barely detectable so we did not use them. The whole track lasted 50 minutes after which the object disappeared from the SALT observing window.

The detection of short period light variations of $2006 \mathrm{RH}_{120}$ prepared as for the next several runs. On 15 March the weather was photometric, and the average $F W H M$ of stellar images was $2^{\prime \prime}$. The asteroid was already brighter than on 11 March but it was also moving faster so we cut the exposure time to $7 \mathrm{~s}$ and used the clear filter to maximize the flux. During the 45 min observation the field of view had to be moved several times to follow the object so there were no common comparison stars for the whole track.

A similar observing procedure (clear filter and $7 \mathrm{~s}$ exposures) were also used on $16 \mathrm{March}$, when 60 min of data were collected. However, there was thin cirrus in the sky and on one occasion a passing cloud made the object invisible. On 17 March the first half of the night was photometric but the image quality was inferior with the average $F W H M$ of stellar images being $3^{\prime \prime}$. A series of $7 \mathrm{~s}$ long exposures through the clear filter was collected during a one hour track.

On 18 March, the last night when $2006 \mathrm{RH}_{120}$ could be observed with SALT, technical problems limited our observations to just three $7 \mathrm{~s}$ long exposures, which were used only for astrometry.

Prior to photometric measurements all CCD frames were pre-reduced with a set of IRAF scripts to remove low level instrumental effects such as bias. Unfortunately it was not possible to apply a flat-field correction. The magnitudes of the asteroid and several comparison stars were measured with circular apertures of $5^{\prime \prime}$ diameter and the estimation of the sky background was obtained from a circular annulus. This part of the reduction was done using the Starlink GAIA program, with the apertures manually placed over the fast moving asteroid to avoid contamination from numerous nearby stars. The correction for light-time was negligible (less than $1 \mathrm{~s}$ ) and was not applied.

The uncertainties in the relative magnitudes of the asteroid are usually best determined from the scatter of the comparison stars of the same brightness. In our case, however, the asteroid was quickly moving across the field of view which was not flatfielded (this introduced systematic effects of up to $0.05 \mathrm{mag}$ ) and in which the scattered light was present (it was subtracted with the background but increased the random noise). While the relative magnitudes of the comparison stars revealed the scatter to be of the level of $0.1 \mathrm{mag}$ we estimate the relative brightness of the asteroid to be accurate to within $0.15 \mathrm{mag}$. Not being able to separate the systematic and random factors of this error we assumed restrictively that all lightcurve points contained Gaussian noise with $\sigma=0.15 \mathrm{mag}$.

Due to shifts of the field of view during the observations, on each night three separate intervals were selected with the same comparison stars. Unfortunately, not all CCD frames were of

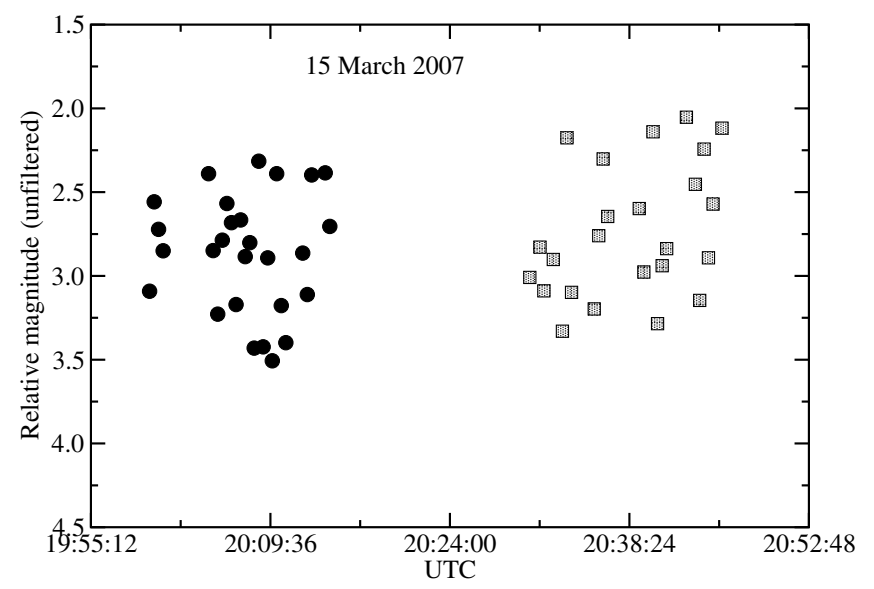

Fig. 1. Lightcurve of $2006 \mathrm{RH}_{120}$ on 15 Mar. 2007. Two subsets marked by different symbols were obtained with different comparison stars and are offset in magnitude.

sufficient quality to be used in further analysis as the asteroid frequently merged with nearby stars and sometimes the image quality deteriorated due to problems with the alignment of the primary mirror segments. Consequently there are gaps in the sequence of measurements and the number of points is less than would result from the $15 \mathrm{~s}$ readout time, separating the $7 \mathrm{~s}$ exposures. For 15 and 16 March we obtained two 15 min long lightcurves and for 17 March only one was of sufficient quality. An example of the lightcurve from 15 March is presented in Fig. 1. The two subsets marked by different symbols are shifted in magnitude. There is no typical quasi-sinusoidal brightness variation in this plot but this often happens when a lightcurve is under-sampled with a frequency comparable to the asteroid rotation frequency.

\section{Rotation period}

To determine a synodical rotation period (or the lack thereof if the object was in a non-principal mode of rotation) we used the method described in Pravec et al. (2000).

The asteroid light variations were approximated by a Fourier series depending on time $t$ and period $P$ :

$$
V(t)=\bar{V}+\sum_{k=1}^{n}\left(A_{k} \sin \frac{2 \pi k\left(t-t_{\mathrm{o}}\right)}{P}+B_{k} \cos \frac{2 \pi k\left(t-t_{\mathrm{o}}\right)}{P}\right)
$$

where $\bar{V}$ is the average brightness and $A_{k}$ and $B_{k}$ are Fourier coefficients. Assuming $P$ to be constant this equation was linearized and solved by least squares. This was repeated for different trial periods ranging from 0.00045 to 0.01 days (from $39 \mathrm{~s}$ to $14.4 \mathrm{~min}$ ). When necessary, a shift in magnitude was also simultaneously searched for to bring the data obtained with different comparison stars to the same level. Calculations were performed with the Fourier series of the 6th or, in case of the evident overfit, of the 4th order. The best fitting parameters were found through the $\chi^{2}$ minimization.

The accuracy of the derived parameters was obtained with the Monte Carlo simulations (Press et al. 1992). The Fourier series approximation was assumed to be the "true" lightcurve, whose points were perturbed thirty times with Gaussian noise with $\sigma=0.15 \mathrm{mag}$. For each of the obtained lightcurves a period was derived as described above. The standard deviation of the obtained set of thirty periods was assumed as an estimation of the accuracy of the result. 


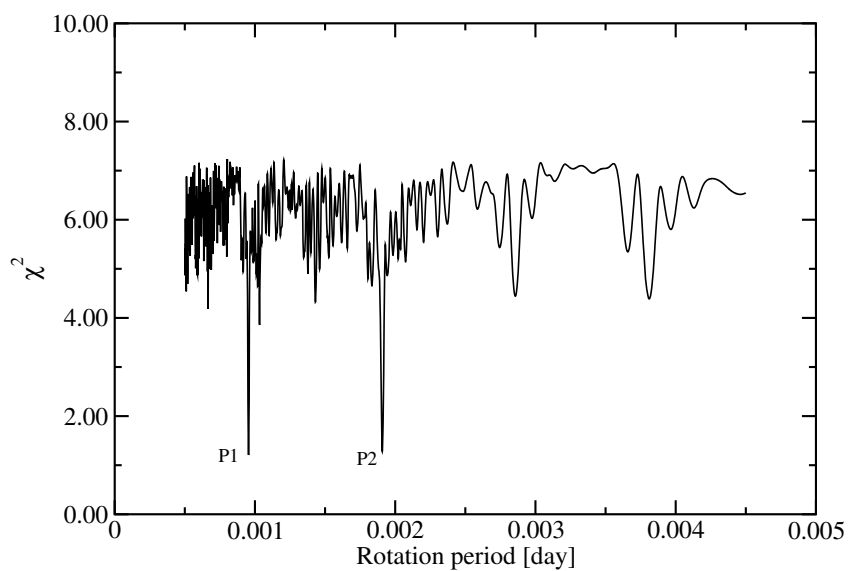

Fig. 2. $\chi^{2}$ plot for various trial periods for the 15 March data from Fig. 1. A shift of $0.12 \mathrm{mag}$, obtained during the least-square fit, was applied for the second subset of points.

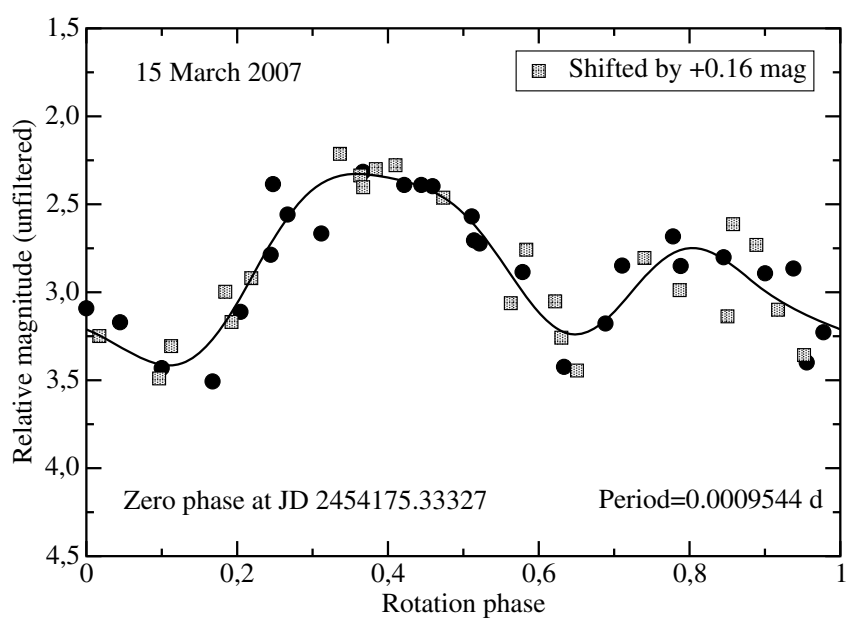

Fig. 3. Composite lightcurve obtained for the data from Fig. 1 with a period $P_{1}=1.3743 \mathrm{~min}$. The best-fit 4 th order Fourier series is plotted as well.

An example plot of the $\chi^{2}$ (per degree of freedom) versus period, obtained with the 4th Fourier series for the lightcurve from Fig. 1 is presented in Fig. 2. There are two minima visible, around 1.37 and $2.75 \mathrm{~min}$. To derive more accurate values for each of them, we repeated calculations around both minima with smaller steps, using both the 4th and the 6th order Fourier series. For the shorter period the 6th order produced an overfit so we stayed with the 4th order solution of $P_{1}=1.3743 \pm 0.0009 \mathrm{~min}$ (Fig. 3 ). In the case of the longer period, the 6th order was able to better approximate the sharp minima (Fig. 4). The period found in this case was $P_{2}=2.7498 \pm 0.0014 \mathrm{~min}$.

The same procedure was repeated for the data from $16 \mathrm{March}$ and resulted in a $\chi^{2}$ plot very similar to that from Fig. 2. There were also two solutions for the period there, at $P_{1}=1.3756 \pm$ $0.0007 \mathrm{~min}$ (from the 4th order fit) and $P_{2}=2.7510 \pm 0.0019 \mathrm{~min}$ (from the 6th order fit), which are quite consistent with those from 15 March. The composite lightcurves in Figs. 5 and 6, obtained with these periods, have similar shapes and amplitudes to those from 15 March.

The Fourier fit to the data from 17 March also yielded two solutions for the synodical period but - due to the smaller number of points from that night - both periods have larger uncertainties. What is more, the composite lightcurve for $P_{1}=$ $1.37 \pm 0.01 \mathrm{~min}$ had its points collected in four tight groups with

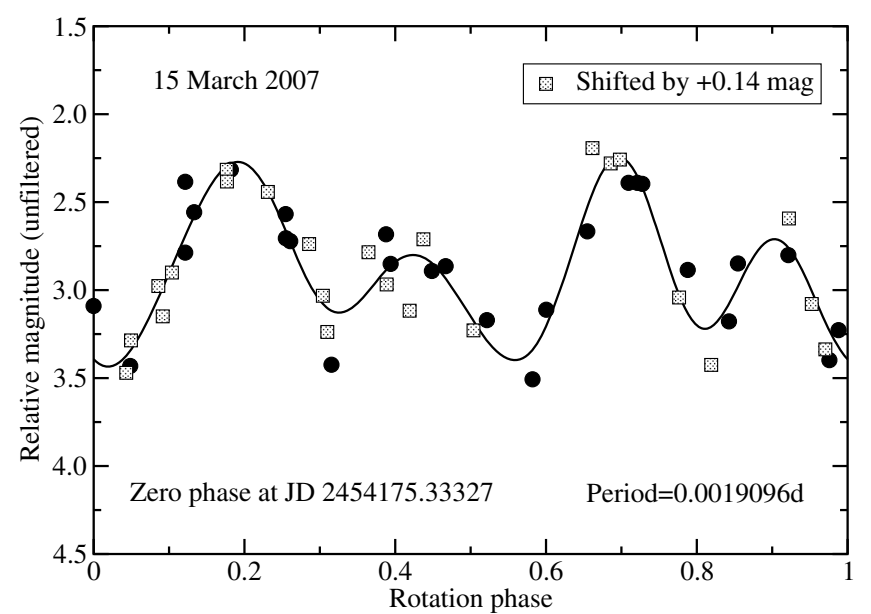

Fig. 4. Composite lightcurve obtained for the data from Fig. 1 with a period $P_{2}=2.7498 \mathrm{~min}$. The best-fit 6 th order Fourier series is plotted as well.

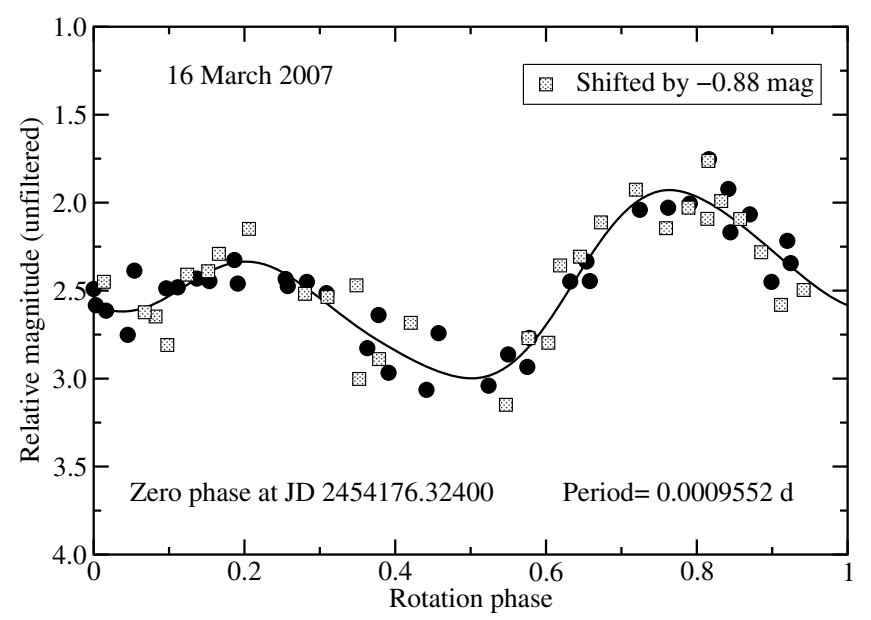

Fig. 5. Composite lightcurve obtained for the data from 16 Mar. with a fitted period $P_{1}=1.3756 \mathrm{~min}$. The best-fit 4 th order Fourier series is also shown.

wide gaps in between so the fitted line looked unreasonable this is why we do not present it here. The lightcurve obtained for $P_{2}=2.72 \pm 0.01 \mathrm{~min}$ is shown in Fig. 7 .

As the position of the asteroid spin vector is not known, we cannot determine the sidereal period of rotation. It is possible, however, to estimate the difference $\Delta P$ between the sidereal and synodic periods. During our observations $2006 \mathrm{RH}_{120}$ moved perpendicularly to the ecliptic and the solar phase angle remained constant. Thus if the asteroid spin had been positioned in the ecliptic plane and at the same time perpendicular to the phase angle bisector, $\Delta P$ would have reached a value of about $0.0001 \mathrm{~min}$, one order of magnitude smaller than the accuracy of our measurements.

The values of $P_{1}$ and $P_{2}$ obtained from 15 and 16 March lightcurves are consistent with each other within their uncertainties. This suggests that the rotation axis of $2006 \mathrm{RH}_{120}$ does not change its position in space at a level we could detect. Because of that, we can safely average the periods obtained on consecutive nights. For that we used only results from 15 and 16 March because the 17 March data are less accurate. The obtained values are: $P_{1}=1.375 \pm 0.001 \mathrm{~min}$ and $P_{2}=2.750 \pm 0.002 \mathrm{~min}$.

There is still a question of whether the accuracy of the obtained periods is sufficent to use the data from two nights in one 


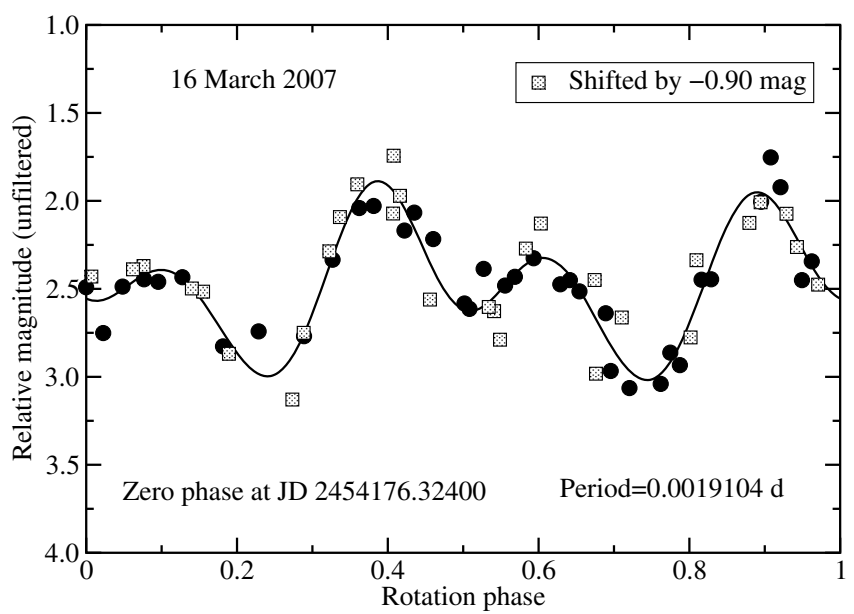

Fig. 6. Composite lightcurve obtained for the data from 16 Mar. with a fitted period $P_{2}=2.7510$ min. The best-fit 6th order Fourier series is superimposed.

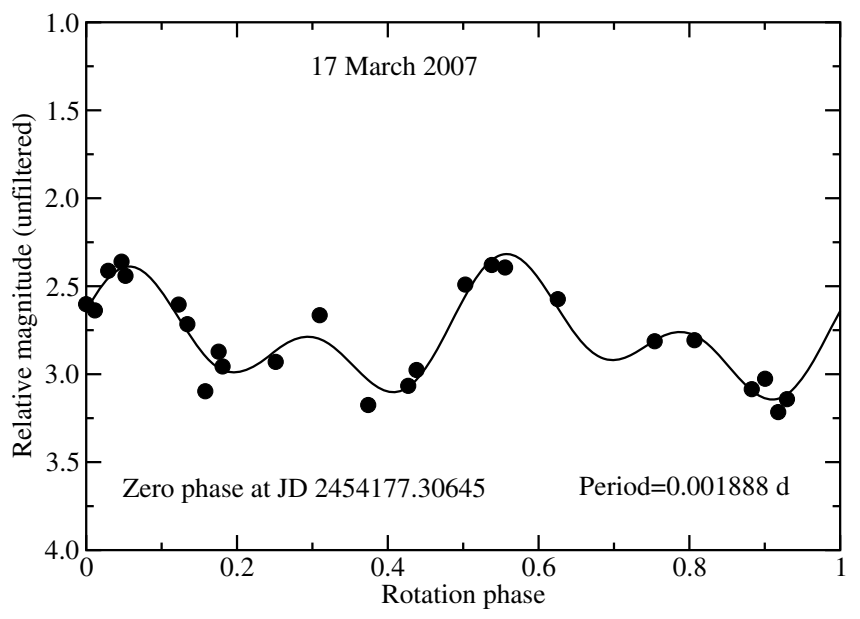

Fig. 7. Composite curve from 17 Mar., folded with a period $P_{2}=$ 2.72 min. The best-fit 4th order Fourier series is also shown.

fit. To check this we performed a similar analysis as before, using simultaneously the lightcurves from 15 and 16 March (the $P_{1}$ solution) with the points already shifted to the same level within each night. The result in the form of a $\chi^{2}$ plot consisted of the regular comb-like structure of minima, all of them at the same level, separated by $0.0014 \mathrm{~min}$. They corresponded to different numbers of full rotations that the asteroid performed between 15 and 16 March - the half-cycle ambiguity was not present because of the asymmetry of the lightcurve extrema in the $P_{1}$ solution. The calculation of the formal uncertainties for the obtained set of minima was difficult given the slightly different shapes of both lightcurves, which introduced systematic effects.

Unfortunately, the uncertainties of the individual solutions from both nights $(0.0007$ and $0.0009 \mathrm{~min}$ for the 15 and 16 March, respectively) were too high to break the ambiguity in the unknown number of cycles between them. On the other hand, the value of $P_{1}=1.375 \pm 0.001 \mathrm{~min}$ obtained by averaging the individual solutions from both nights was very close to one of the minima on the $\chi^{2}$ plot $(1.3749 \mathrm{~min})$. A similar ambiguity was encountered when trying to combine lightcurves from both nights in case of the $P_{2}$ solution.

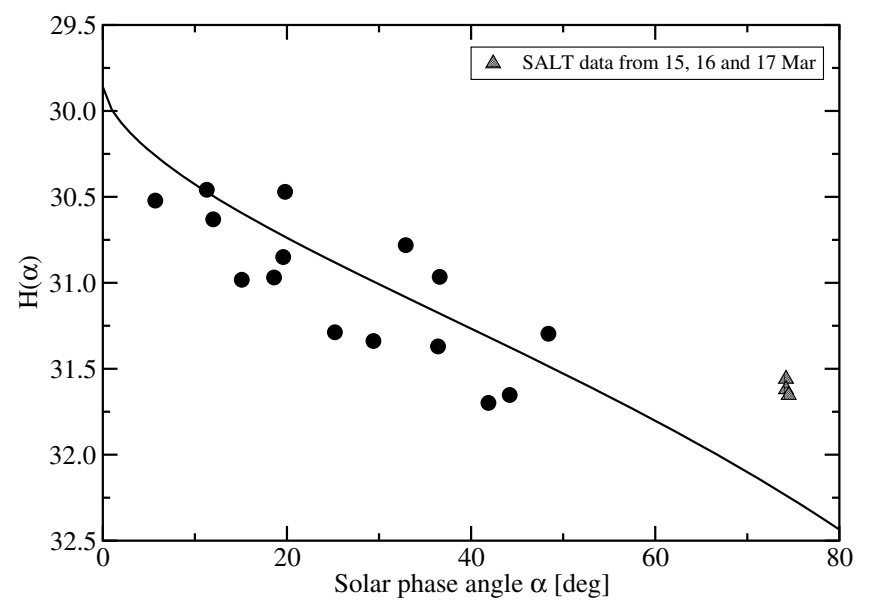

Fig. 8. Phase curve for $2006 \mathrm{RH}_{120}$, obtained with selected observations reported to the MPC. The best fit was derived for $H=29.9 \mathrm{mag}$, while $G$ was kept at 0.25 .

\section{Size and shape}

The absolute magnitude of $2006 \mathrm{RH}_{120}$, as given by the Minor Planet Center (Bressi et al. 2008), is $H=29.5$ mag. It was determined in a standard way (assuming $G=0.15$ ) from all available brightness measurements, obtained as a by-product during the astrometric observations. Some of them, however, were done with small telescopes and their accuracy was very low. To obtain a better estimate of $H$ we selected results only from larger instruments (including SALT), then averaged magnitudes obtained on a single night and discarded the evident outliers. Results are presented in Fig. 8 with the superimposed $H-G$ curve, obtained from a least-square fit. Due to the significant scatter of the data, we did not fit the $G$ parameter which was kept constant at 0.25 (this is an average value used for the S-type asteroids, Bowell et al. 1989). The $H$ parameter obtained from the fit is $29.9 \mathrm{mag}$.

A large scatter of the data points about the fitted curve is caused by several factors: low signal-to-noise of the asteroid images, systematic errors in the magnitude scale zero point, biases in the average magnitudes resulting from sparse sampling of the rotation period and the systematic effect caused by the changing geometry of observation/illumination. The last effect is most probably responsible for the large deviation of the three SALT measurements (which are quite consistent among themselves) from the fitted curve.

Being unable to model all sources of inaccuracies that influenced the brightness measurements, we assumed rather conservative upper and lower bounds for the $H$ parameter: 29.6 mag and 30.2 mag. As about $70 \%$ of the observed near-Earth asteroids are S- and Q-type with the average geometric albedo $p_{V}=0.18$ (Binzel et al. 2002) we can use this value for an estimate of the $2006 \mathrm{RH}_{120}$ effective diameter $D$. Using the standard formula: $D=1329 \times 10^{(-H / 5)} \times p_{V}^{(-1 / 2)}$ from Fowler \& Chillemi (1992), we obtain $D=3.3 \pm 0.4 \mathrm{~m}$. Of course, there are nearEarth asteroids which are darker $\left(p_{V}=0.06\right.$ and $G=0.15$ for C-type objects) or brighter ( $p_{V}=0.30$ and $G=0.40$ for V-type objects), (Bowell et al. 1989). For them, repeating the same calculations as before, we get, for the low albedo objects $H=29.4-30.0 \mathrm{mag}, D=5.4-7.2 \mathrm{~m}$, and for the high albedo objects $H=29.7-30.3 \mathrm{mag}, D=2.1-2.8 \mathrm{~m}$. To sum up, the diameter of $2006 \mathrm{RH}_{120}$ lies in the interval $2 \mathrm{~m}<D<7 \mathrm{~m}$, with the most probable value of $3.3 \pm 0.4 \mathrm{~m}$.

Without knowing the pole position it is impossible to derive a triaxial approximation of the asteroid shape. We can, 
however, estimate its $a / b$ elongation assuming that the amplitude of $1.2 \mathrm{mag}$ is the maximum that can be observed at $\alpha=74^{\circ}$. A classical approach to reduce the observed amplitude to a zero phase angle and then use the triaxial ellipsoid approximation to derive $a / b$ could not be used here because of the influence of obliquity (Gutierrez et al. 2006). Instead, we used the algorithm of Kwiatkowski (1995) to generate a large set of model lightcurves for different triaxial ellipsoid shapes and pole positions, assuming the observing/illumination geometries of 15-17 March 2007. Next we compared the obtained model amplitudes with the observed ones trying to find best matches. Unfortunately, no simultaneous fit for the three amplitudes was found which, at such high phase angle, is not surprising. The model shapes that fit individual lightcurves, however, had all elongations $a / b \geq 1.4$ and this value can be accepted as the estimate of the asteroid overall elongation.

\section{Origin}

$2006 \mathrm{RH}_{120}$ is a member of a small group of asteroids which move on Earth-like orbits (hereafter ELO). They were recently studied by Brasser \& Wiegert (2008), who define them as objects whose orbital parameters satisfy the criteria: $a \in$ $[0.95,1.05] \mathrm{AU}, e \in[0,0.1], i \in\left[0^{\circ}, 10^{\circ}\right]$ and at the same time are not Earth co-orbitals. These intervals were chosen arbitrarily and, as the authors admit, there are asteroids just outside these limits that also have Earth-like orbits. They mention the possibility of using the selection criterion $U<0.1$ or $U<0.15$ (where $U$ is a geocentric velocity, see next paragraph). This, however, would have a side-effect of including objects on distinctly non Earth-like orbits and was not used. Within the accepted criteria Brasser \& Wiegert (2008) found 13 ELO asteroids (as of 3 Jun. 006). We should warn the reader about errors in the last two rows of Table 1 in Brasser \& Wiegert (2008), where incorrect values of $T_{\mathrm{E}}$ and $U$ were given for $2006 \mathrm{JY}_{26}$ and $2005 \mathrm{CN}_{61}$.

Let us now see how the orbit of $2006 \mathrm{RH}_{120}$ compares with orbits of other known ELO asteroids. Using the NASA Horizons System (http://ssd.jpl.nasa.gov/?horizons) we found 17 objects on ELOs (as of 1 Sep. 2008), excluding $2003 \mathrm{YN}_{107}$ which is an Earth co-orbital (Connors et al. 2004). To display their mutual orientation we used two coordinates: $U$ and $\cos \theta$. $U$ is the dimensionless encounter velocity with respect to the Earth at infinity: $U=\sqrt{3-T_{\mathrm{E}}}$, where $T_{\mathrm{E}}$ is the Tisserand parameter related to the Earth. The second parameter, $\theta$, is the angle between the vector of the orbital velocity of the Earth and the vector of geocentric velocity of the asteroid (Valsecchi et al. 1999). $T_{\mathrm{E}}$ (and hence, $U$ ) are rather well conserved at close encounters with the planet (Carusi et al. 1995) contrary to $a, e$ and $i$ which, in the case of ELO asteroids, can chaotically change during each passage close to the Earth (Brasser \& Wiegert 2008). The $U$ and $\cos \theta$ are successfully used for meteoroid stream identification Valsecchi et al. 1999.

Contrary to the rest of asteroids, for $2006 \mathrm{RH}_{120}$ we chose the pre-encounter orbital parameters $(a=0.9533 \mathrm{AU}, e=0.0505$ and $i=0.5663$ ) because after the Earth encounter its orbit became a non-crossing one and the $U$ parameter was undefined. For comparison, we also added the orbit of J002E3, whose origin is already known (the data come from the NASA Horizons System). The $U$ and $\cos \theta$ for all these objects are presented in Fig. 9. The value of $\cos \theta$ close to -1 in the case of $2006 \mathrm{RH}_{120}$ and J002E3 implies they were approached by the Earth from behind. When close to the planet, they entered its Hill sphere through the inner $L_{1}$ Lagrange point. This plot, however, is a

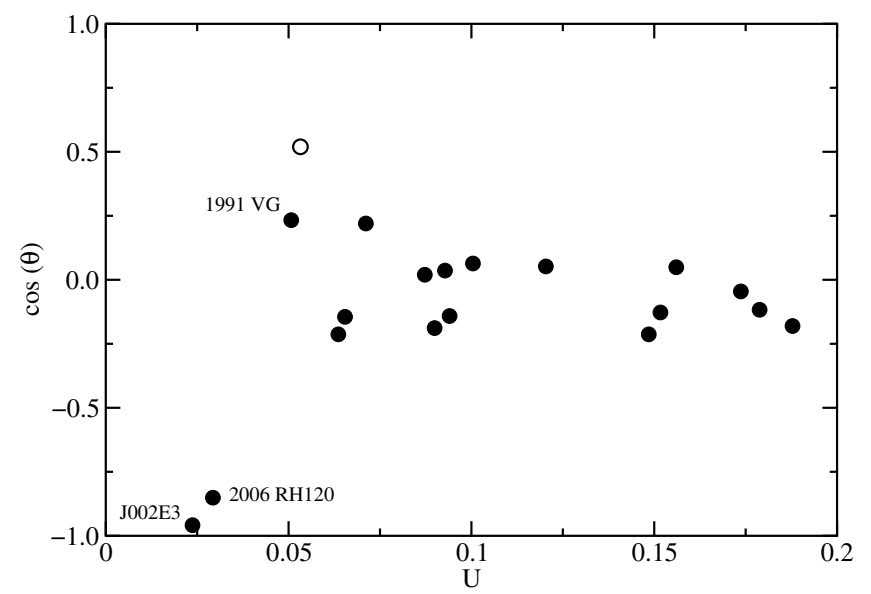

Fig. 9. Orbits of all known ELO asteroids in the $U, \cos \theta$ coordinates. The three objects discussed in the paper: 1991 VG1, J002E3 and $2006 \mathrm{RH}_{120}$ are indicated. The empty circle represents the orbit of 1991 VG1 in 1825.

snapshot of a dynamic evolution of the orbits, whose range can be illustrated by the empty circle in Fig. 9. It represents the orbit of 1991 VG1 in 1825 (it was obtained from digitized plots showing the changes of $a, e, i$ elements of this asteroid in Brasser \& Wiegert 2008).

The orbital evolution of comparable scale happens to other ELO asteroids so a thousand years ago $2006 \mathrm{RH}_{120}$ could have been much closer (on the $U, \cos \theta$ plot) to the other ELO asteroids. Also, with new ELO objects being discovered in the future, the gap in the plot can be filled. It is thus very likely $2006 \mathrm{RH}_{120}$ can have the same origin as the other ELO asteroids that come from low-eccentricity Amor and Apollo orbits (Bottke et al. 1996; Brasser \& Wiegert 2008). In this scenario, after being perturbed into the Earth-like orbit, the $U$ velocity of $2006 \mathrm{RH}_{120}$ may have decreased to the present value of 0.03 by repeated passages close to the Earth. It would be worth the effort to follow the orbital evolution of $2006 \mathrm{RH}_{120}$ numerically as far back as the chaotic nature of the Earth's perturbations allow.

With this hypothesis as the most probable, let us now consider the possibility that $2006 \mathrm{RH}_{120}$ was ejected from the Moon. Brasser \& Wiegert (2008) discussed this possibility for a general population of ELO asteroids, while here we will focus specifically on $2006 \mathrm{RH}_{120}$. We estimate the probability of the ejection of a $D_{\mathrm{e}}=3 \mathrm{~m}$ rock from the lunar surface during the cratering impact.

The size-velocity distribution for the ejecta in the case of impacts can be derived from laboratory experiments (Nakamura et al. 1994), numerical simulations (Nolan et al. 1996) or studies of craters on the Moon (Vickery 1987) and Mars (Hirase et al. 2004). The ejecta diameter $D_{\mathrm{e}}$ is usually normalized by the projectile radius, $R_{\mathrm{p}}$ and plotted versus the ejection velocity. A compilation of results from different sources was presented by Hirase et al. (2004) for the gravity regime in which the material strength can be rejected. For the Moon this last condition is true for craters with diameters larger than $600 \mathrm{~m}$ (McEwen et al. 2005). The data in Fig. 4 of Hirase et al. (2004) extend only to the ejection velocity of $1 \mathrm{~km} \mathrm{~s}^{-1}$ which is smaller than the escape velocity for the Moon $\left(2.4 \mathrm{~km} \mathrm{~s}^{-1}\right)$. The theoretical model of Melosh (1984) allows predictions of ejection velocities of $10 \mathrm{~km} \mathrm{~s}^{-1}$ but considers results beyond $1 \mathrm{~km} \mathrm{~s}^{-1}$ uncertain. However, Preblich et al. (2007), who studied secondary craters of Zunil on Mars, shows that this relation can be extended at least to $2.2 \mathrm{~km} \mathrm{~s}^{-1}$ and probably even to $3.5 \mathrm{~km} \mathrm{~s}^{-1}$. If we extrapolate 
the lunar data in Fig. 4 of Hirase et al. (2004) to $2.4 \mathrm{~km} \mathrm{~s}^{-1}$ we find that ejecta blocks of the size $D_{\mathrm{e}} / R_{\mathrm{p}}<0.01$ can escape from the Moon. Thus, to eject the $D_{\mathrm{e}}=3$ m body from the Moon, an impact of the projectile of the diameter $D_{\mathrm{p}}>600 \mathrm{~m}$ is required. Such a body would create a crater of the diameter of $D_{\mathrm{c}}=10 \mathrm{~km}$ (Eq. (6) in Bottke et al. 2000) which confirms we are well inside the gravity regime.

From the impactor size-frequency distribution for the Moon (Werner et al. 2002) we can estimate that the impact of the $D_{\mathrm{p}}=600 \mathrm{~m}$ asteroid on the Moon happens with a probability smaller than $2 \times 10^{-7} \approx 10^{-7}$ per year. Given the estimated life-time of objects on ELOs of $10^{4}$ years (Brasser \& Wiegert 2008), a chance to observe $3 \mathrm{~m}$ lunar ejecta on such an orbit is low $\left(10^{-3}\right)$. What is more, the lunar meteorites, half of which fell to the Earth during the last $10^{5}$ years, favour the "small impact" scenario according to which they were ejected during several impacts of projectiles smaller than 10-30 m (Head 2001; Artemieva \& Ivanov 2004). As a result, the hypothesis of the lunar origin of $2006 \mathrm{RH}_{120}$ and other ELO asteroids larger than several metres can be rejected.

The case of cometary nuclei impacting the Moon is not considered separately as their input to the projectile flux is secondary (Werner et al. 2002). Also, even though their impact velocities are greater $\left(\sim 55 \mathrm{~km} \mathrm{~s}^{-1}\right.$ for parabolic comets versus $\sim 18 \mathrm{~km} \mathrm{~s}^{-1}$ for the asteroids, Steel 1998), their bulk densities are about ten times smaller and the result of an impact is thus comparable to that of an asteroid (for a given diameter of the lunar crater, $R_{\mathrm{p}} \sim\left(\rho_{\mathrm{t}} / \rho_{\mathrm{p}}\right)^{0.43} \times v_{\mathrm{p}}^{-0.56}$, where $\rho_{\mathrm{t}}$ and $\rho_{\mathrm{p}}$ are the densities of the target and the projectile and $v_{\mathrm{p}}$ is the velocity of the projectile; Bottke et al. 2000).

There is also another mechanism for transferring metre-sized near-Earth asteroids to the Earth-like orbits worth considering. We know of meteoroids that traveled through the Earth's atmosphere and returned to space at a reduced velocity. The best known is the fireball of 10 August 1972 (Ceplecha 1994), with the estimated diameter of several metres, but even more interesting is the 3 October 1996 bolid (Hills \& Goda 1997). It was seen over New Mexico and Texas and 100 minutes later another fireball was observed above California. There is a chance that those two were the same object that approached the Earth at a height of $35-40 \mathrm{~km}$, orbited it once and plunged to the ground.

The possibility of gravitational capture of grazing meteoroids was discussed by Hills \& Goda (1997). They considered both the stony and nickel-iron meteoroids that enter the atmosphere at the height of $100 \mathrm{~km}$ above the Earth. Calculations were performed for sizes of 1-20 m and for impact velocities at infinity $v_{\infty}=5-20 \mathrm{~km} \mathrm{~s}^{-1}$. Assuming the average impact velocity with the Earth of small asteroids $v_{\mathrm{i}}=20 \mathrm{~km} \mathrm{~s}^{-1}$ (Steel 1998; Brown et al. 2002) we obtain $v_{\infty}$ as $\sqrt{v_{\mathrm{i}}^{2}-v_{\mathrm{E}}^{2}} \approx 17 \mathrm{~km} \mathrm{~s}^{-1}$, where $v_{\mathrm{E}}=11.2 \mathrm{~km} \mathrm{~s}^{-1}$ is the Earth's escape velocity. According to Hills \& Goda (1997), for this value of $v_{\infty}$ the fraction of iron impactors captured to the total number that collide with the planet is $5 \times 10^{-4}$ for all considered diameters. For stony objects the percentage is the same but only for diameters of 1 to $5 \mathrm{~m}$. Meteoroids with larger diameter fragment in the atmosphere which greatly increases the atmospheric drag and makes them fall to the ground.

The flux of small near-Earth asteroids colliding with the Earth is estimated to be 10 of $1-5$ m objects per year (Brown et al. 2002) which means every 200 years one such object can potentially be captured into a bound orbit. Unfortunately, the semimajor axis of such an orbit is of the order of $10^{4} \mathrm{~km}$ which means the meteoroid plunges to the ground on its second trip through the atmosphere.

The situation is different if $v_{\mathrm{i}}<14 \mathrm{~km} \mathrm{~s}^{-1}\left(v_{\infty}<7 \mathrm{~km} \mathrm{~s}^{-1}\right)$. According to Hills \& Goda (1997), in such case the fraction of captured meteoroids is $1.5 \times 10^{-3}$ and roughly one fourth of them end up on orbits with a semimajor axes $a>10^{5} \mathrm{~km}$. Depending on the orientation of such orbits in space, there is a possibility that the perturbations from the Moon and/or the Sun can then raise their perigee above the atmosphere so that they can make several revolutions about the Earth before they impact the Earth, the Moon or are transferred to the heliocentric orbit. This scenario is possible for about $3 \times 10^{-4}$ of all objects with $v_{\mathrm{i}}<14 \mathrm{~km} \mathrm{~s}^{-1}$ impacting the Earth. According to Steel (1998), 25\% of Earth crossing asteroids have impact speed $v_{\mathrm{i}}<13.8 \mathrm{~km} \mathrm{~s}^{-1}$ so the final probability of the temporary satellite capture is then $3 \times 10^{-4} \times 0.25 \times 10 / \mathrm{yr} \approx 10^{-3} / \mathrm{yr}$. Again, when we compare this to the average time the asteroid can spend on the ELO $\left(10^{4}\right.$ years $)$ we see we cannot neglect the possibility that $2006 \mathrm{RH}_{120}$ went through an episode of aerobraking in the Earth's atmosphere. If true, this event might have launched it on a heliocentric orbit very similar to the one it was discovered on. Clearly, this possibility needs further investigation.

\section{Conclusions}

The most interesting thing about $2006 \mathrm{RH}_{120}$ is its heliocentric orbit which brought it into the Earth's Hill sphere, where it spent a year orbiting the planet. It is also one of the smallest objects observed so far and the smallest one for which photometric and radar data were collected ${ }^{1}$. From the latter we can learn it is a natural body (Hergenrother et al, in preparation) and not space debris left from one of the planetary missions.

The rotation period of $2006 \mathrm{RH}_{120}$ was found to be either $P_{1}=1.375 \pm 0.001$ or $P_{2}=2.750 \pm 0.002 \mathrm{~min}$. The lightcurve amplitude of $1.2 \mathrm{mag}$ observed at the solar phase angle $\alpha=74^{\circ}$ suggests a substantial elongation of this body with the $a / b \geq 1.4$. The phase curve fitted to the observed average brightnesses gives the absolute magnitude $H=29.9 \pm 0.3$ mag which translates into an effective diameter of $D=3.3 \pm 0.4 \mathrm{~m}$ for the most typical near-Earth asteroid albedo. If we consider darker and brighter asteroids as well, then its diameter lies in the interval $2 \mathrm{~m}<$ $D<7 \mathrm{~m}$. In the NASA Horizons database there are only eight asteroids (as of 1 Sep. 2008) with comparable diameters (with $H>29.5$ mag).

Being on an orbit similar to that of the Earth, $2006 \mathrm{RH}_{120}$ could come from the low eccentricity Apollo or Amor orbit. This scenario has been studied in detail by Brasser \& Wiegert (2008) who concluded there can be several hundreds of $10 \mathrm{~m}$ sized bodies in the ELOs which can be observed only for a short time during their long synodic periods of about 25 years.

While the lunar origin of $2006 \mathrm{RH}_{120}$ can be excluded, there is also a possibility that it was a typical near-Earth asteroid that lost some of its kinetic energy in the Earth's atmosphere and was then redirected, by luni-solar perturbations, to the heliocentric Earth-like orbit. This possibility should still be checked as no detailed simulation of such a trajectory has been performed so far. If true, such an episode would leave its mark on the asteroid surface in the form of a dark ablation crust which could appear in its spectra.

Acknowledgements. The authors wish to express their gratitude for valuable discussions to G. Tancredi, B. Gladman and T. Jopek. We would also like to thank

\footnotetext{
1 After the submission of this paper photometric data were obtained for an even smaller asteroid, $2008 \mathrm{TC} 3(H=30.7 \mathrm{mag})$.
} 
P. Kankiewicz for his astrometric analysis of our observations which resulted in the improved orbit of $2006 \mathrm{RH}_{120}$ and J. Durech for his helpful comments and suggestions as a reviewer. The first author acknowledges support from the Polish MNiI/MNiSzW Grant 1 P03D 02529.

All of the observations reported in this paper were obtained with the Southern African Large Telescope (SALT), a consortium consisting of the National Research Foundation of South Africa, Nicholas Copernicus Astronomical Center of the Polish Academy of Sciences, Hobby Eberly Telescope Founding Institutions, Rutgers University, Georg-August-Universitat Gottingen, University of Wisconsin-Madison, Carnegie Mellon University, University of Canterbury, United Kingdom SALT Consortium, University of North CarolinaChapel Hill, Dartmouth College, American Museum of Natural History and the Inter-University Centre for Astronomy and Astrophysics, India.

\section{References}

Artemieva, N., \& Ivanov, B. 2004, Icarus, 171, 84

Binzel, R. P., Lupishko, D., di Martino, M., Whiteley, R. J., \& Hahn, G. J. 2002, in Asteroids III, ed. W. F. Bottke Jr., A. Cellino, P. Paolicchi, \& R. P. Binzel (Univ. of Arizona Press), 255

Bottke, W. F., Nolan, M. C., Melosh, H. J., Vickery, A. M., \& Greenberg, R. 1996, Icarus, 122, 406

Bottke, W. F., Love, S. G., Tytell, D., \& Glotch, T. 2000, Icarus, 145, 108

Bowell, E., Hapke, B., Domingue, D., et al. 1989, in Asteroids II, ed. R. Binzel, T. Gehrels, \& M. Matthews (Univ. of Arizona Press), 524

Brasser, R., \& Wiegert, P. 2008, MNRAS, 386, 2031

Bressi, T. H., Hergenrother, C. W., Christensen, E. J., et al. 2008, Minor Planet Electronic Circular, D12

Brown, P., Spalding, R. E., ReVelle, D. O., Tagliaferri, E., \& Worden, S. P. 2002, Nature, 420, 294

Buckley, D. A. H. 2001, New Astron. Rev., 45, 13

Buckley, D. A. H., Swart, G. P., \& Meiring, J. G. 2006, in Society of PhotoOptical Instrumentation Engineers (SPIE), Conf. Ser., 6267, 32

Carusi, A., Kresak, L., \& Valsecchi, G. B. 1995, Earth Moon and Planets, 68, 71

Ceplecha, Z. 1994, A\&A, 283, 287
Connors, M., Veillet, C., Brasser, R., et al. 2004, in Lunar and Planetary Institute Conference Abstracts, 35, 1565

Fowler, J. W., \& Chillemi, J. 1992, in The IRAS Minor Planet Survey, ed. E. Tedesco, G. Veeder, J. Fowler, \& J. Chillemi (Hanscom Air Force Base, MA: Phillips Laboratory), 17

Gutierrez, P. J., Davidsson, B. J. R., Ortiz, J. L., Rodrigo, R., \& Vidal-Nunez, M. J. 2006, A\&A, 454, 367

Head, J. N. 2001, in Lunar and Planetary Institute Conference Abstracts, 32, 1768

Hills, J. G., \& Goda, M. P. 1997, Planet. Space Sci., 45, 595

Hirase, Y., Nakamura, A. M., \& Michikami, T. 2004, Planet. Space Sci., 52, 1103

Jorgensen, K., Rivkin, A., Binzel, R., et al. 2003, BAAS, 35, 981

Kwiatkowski, T. 1995, A\&A, 294, 274

Lambert, J., Hamada, K., Hall, D., et al. 2004, in Proceedings of the 2004 IEEE Aerospace Conference, 5, 2866

McEwen, A. S., Preblich, B. S., Turtle, E. P., et al. 2005, Icarus, 176, 351

Melosh, H. J. 1984, Icarus, 59, 234

Mikkola, S., Innanen, K., Wiegert, P., Connors, M., \& Brasser, R. 2006, MNRAS, 369, 15

Nakamura, A. M., Fujiwara, A., \& Kadono, T. 1994, Planet. Space Sci., 42, 1043 Nolan, M. C., Asphaug, E., Melosh, H. J., \& Greenberg, R. 1996, Icarus, 124, 359

O’Donoghue, D., Buckley, D. A. H., Balona, L. A., et al. 2006, MNRAS, 372, 151

Pravec, P., Hergenrother, C., Whiteley, R., et al. 2000, Icarus, 147, 477

Preblich, B. S., McEwen, A. S., \& Studer, D. M. 2007, J. Geophys. Res. (Planets), 112, 1

Press, W. H., Vetterling, W. T., Teukolsky, S. A., \& Flannery, B. P. 1992, Numerical Recipes in C (Cambridge University Press)

Ramsey, L. W., Adams, M. T., Barnes, T. G., et al. 1998, in Society of PhotoOptical Instrumentation Engineers (SPIE) Conf. Ser., 3352, 34

Steel, D. 1998, Planetary and Space Science, 46, 473

Tancredi, G. 1998, Celest. Mech. Dyn. Astron., 69, 119

Valsecchi, G. B., Jopek, T. J., \& Froeschle, C. 1999, MNRAS, 304, 743

Vickery, A. M. 1987, Geophys. Res. Lett., 14, 726

Werner, S. C., Harris, A. W., Neukum, G., \& Ivanov, B. A. 2002, Icarus, 156, 287 